\title{
A teoria da equidade reversa se aplica na atenção primária à saúde? Evidências de 5564 municípios brasileiros
}

\author{
Raphael Mendonça Guimarães ${ }^{1}$
}

Como citar Guimarães RM. A teoria da equidade reversa se aplica na atenção primária à saúde? Evidências de 5564 municípios brasileiros. Rev Panam Salud Publica. 2018;42:128 https:/ /doi.org/10.26633/RPSP.2018.128

RESUMO Objetivo. Investigar a evolução da cobertura da atenção primária à saúde (APS) nos municípios brasileiros de 2007 a 2016, no contexto da teoria da equidade reversa.

Métodos. Estudo ecológico realizado com dados de 5564 municípios brasileiros. Os dados foram obtidos do Censo de 2010 e do sistema DATASUS. Os municípios foram classificados quanto ao seu desenvolvimento a partir de indicadores sociais selecionados, através de análise de cluster. Analisou-se a cobertura pela Estratégia Saúde da Família (ESF) nos grupos criados através da média e distância interquartílica, por análise de séries temporais e correlação entre variáveis.

Resultados. Foram criados dois agrupamentos de municípios, de condições mais $(n=3293)$ ou menos favoráveis $(n=2271)$. A diferença entre os grupos, para todos os indicadores avaliados, foi significativa $(\mathrm{P}<0,001)$. Em geral, independentemente do grupo, houve aumento na cobertura da ESF ao longo do período. Contudo, a partir de 2009, a cobertura média da ESF passou a ser maior no grupo com condições menos favoráveis. O aumento na cobertura também foi mais acelerado nesse grupo. Finalmente, uma mudança na correlação entre os indicadores e a cobertura de APS no primeiro e no último ano da série histórica indica que os critérios utilizados para expansão da cobertura no grupo com condições menos favoráveis passaram a valorizar a pobreza absoluta e não a iniquidade.

Conclusões. A APS no Brasil cumpre seu papel de politica redutora de desigualdade de acesso. Portanto, não se aplicou a este caso a teoria da equidade reversa.

Palavras-chave Atenção primária à saúde; políticas públicas; equidade; iniquidade em saúde; Brasil.

A Conferência Internacional de AlmaAta, em 1978, propôs a atenção primária à saúde (APS) como opção prioritária para operacionalizar os serviços de saúde, independentemente do sistema de saúde adotado (1). Desde então, vários

Fundação Oswaldo Cruz (Fiocruz), Escola Politécnica de Saúde Joaquim Venâncio, Rio de Janeiro (RJ), Brasil. Correspondência: raphael. guimaraes@fiocruz.br países desenvolvidos, a exemplo de Canadá e Reino Unido, assumiram o papel da APS, centrada no cuidado médico, como porta de entrada dos sistemas de saúde. Por sua vez, muitos países de economia periférica implementaram uma construção reducionista de APS, que privilegiou, como forma de limitar os gastos em saúde, populações de baixa renda e programas específicos, em formato de cestas básicas de serviços que incluíram áreas consideradas prioritárias, como a saúde materno-infantil (2).

No Brasil, a construção da APS se deu de forma particular, uma vez que, mesmo sendo de economia periférica, o país optou por definir, ao longo do tempo, uma política nacional que orientasse a atenção básica, em substituição ao modelo mais tradicional de programas de saúde (3). Ao longo dos últimos 30 anos, após a promulgação da Constituição de 
1988, houve um processo de transformação das políticas públicas, induzindo a construção da saúde como um direito social (4). Nesse aspecto, o Brasil é reconhecido por uma atuação bem-sucedida no desenvolvimento da APS, especialmente em nível local (5).

Um dos efeitos de período mais importantes desse processo foi a criação do Programa Saúde da Família, em 1994, implementado a partir de 1997, que trouxe consigo um princípio de modelo de assistência centrado nas famílias, operacionalizado nos municípios e contando com tecnologia leve de alta capacidade resolutiva (2). Desde então, o poder executivo federal tem induzido a adoção de políticas públicas que descentralizem as práticas de APS, atribuindo ao nível municipal o papel de cogestor financeiro e organizador da oferta de serviços de saúde, garantindo sua qualidade. Esse movimento tem fortalecido a APS, em oposição a um modelo tradicional hospitalocêntrico, individual, de elevado custo e baixa resolutividade (6). Além disso, esse movimento repercutiu na criação, em 2006, da Política Nacional de Atenção Básica (PNAB), que, entre outros aspectos organizativos, passou a denominar a saúde da família não mais como programa, mas como estratégia, definindo uma prática de substituição progressiva de toda a atenção básica tradicional para o novo modelo $(7,8)$. Nesse contexto, pode-se afirmar que a APS é reconhecida como porta de entrada preferencial do sistema de saúde no Brasil (9).

Ao longo dos anos, a PNAB avançou na promoção do acesso da população mais vulnerável à APS. O impacto da revisão mais recente da PNAB, de setembro de 2017 (10), ainda não pode ser avaliado; porém, merece destaque a edição de 2011 - momento da criação de incentivos para o trabalho em municípios com dificuldade de atração ou retenção de médicos bem como da criação dos Núcleos de Apoio à Saúde da Família (NASF) (11). A edição de 2011 procurou sanar limitações da versão de 2006 no sentido de garantir satisfatoriamente a equidade de acesso aos vulneráveis. Esse padrão está de acordo com a "hipótese da equidade reversa", apresentada por Victora et al. (12). Segundo esses autores, novas intervenções e programas em saúde pública são, inicialmente, mais acessíveis e utilizados por pessoas com situação econômica mais favorável, aumentando as desigualdades socioeconômicas em saúde. Ao longo do tempo, essa lacuna social é estreitada, à medida que a nova tecnologia ou política é alcançável pela população com situação econômica inferior, o que geralmente ocorre quando o impacto da intervenção nos grupos mais ricos já atingiu o seu limite (12).

Nesse sentido, o objetivo do presente estudo é descrever a evolução temporal da cobertura da APS nos municípios brasileiros de 2007 a 2016, no contexto da teoria da equidade reversa.

\section{MATERIAIS E MÉTODOS}

Trata-se de estudo ecológico realizado com dados de 5564 municípios brasileiros no período de 2007 a 2016. Ressalta-se que, devido à mudança no número de municípios ao longo do período analisado, foram considerados somente os municípios existentes durante todo o período de análise. É importante mencionar ainda que o estudo optou por avaliar a APS considerando a cobertura pela ESF. Admite-se a limitação de que, em determinadas unidades de atenção básica que não pertencem à Estratégia, o processo de trabalho, correspondente à atenção primária tradicional, não possui os mesmos pressupostos introduzidos pela PNAB.

Foram utilizados dados provenientes do Censo de 2010, disponibilizados pelo Instituto Brasileiro de Geografia e Estatística (IBGE), para a análise dos indicadores sociais (13). Para os dados referentes à cobertura populacional pela (ESF), foram utilizados dados disponibilizados pelo DATASUS (http://tabnet.datasus.gov. br/cgi/deftohtm.exe?pacto/2015/cnv/ coapmunbr.def). As bases foram compatibilizadas pela técnica de linkage.

Os municípios foram classificados quanto ao seu desenvolvimento. Para tal, foi utilizada análise multivariada por clusters, ou agrupamento, pela abordagem two-step (14). Essa técnica permite a identificação de padrões entre unidades de análise (municípios) que possuam características homogêneas entre si e heterogêneas em relação aos demais grupos $(15,16)$. A abordagem two-step é mais adequada a grandes bases de dados, pois reduz o problema de escalonamento criado nas técnicas hierárquicas.

Sete indicadores sociais foram utilizados para a construção dos agrupamentos: a) taxa de mortalidade infantil, estimada pelo número de óbitos de crianças menores de 1 ano a cada 1000 crianças nascidas vivas; b) razão de dependência, medida pela razão entre o número de pessoas menores de 15 anos e com 65 anos e mais (que caracterizam população dependente) e o número de pessoas com idade de 15 a 64 anos; c) índice de Gini, que mede o grau de desigualdade na distribuição da renda domiciliar per capita - seu valor varia de zero a 1 e, quanto menor, maior a desigualdade; d) proporção de vulneráveis à pobreza, estimada pela proporção de indivíduos com renda per capita domiciliar inferior a meio salário mínimo; e) razão $10 \%$ mais ricos $/ 40 \%$ mais pobres, que estima o grau de desigualdade na distribuição de indivíduos de acordo com a renda domiciliar per capita, ao comparar a renda média dos $10 \%$ mais ricos com a dos $40 \%$ mais pobres; f) índice de Theil-L, uma medida de desigualdade de renda domiciliar per capita, dado que a renda não é nula (portanto, uma medida diferente do índice de Gini) - varia de zero a infinito e, quanto maior, maior a desigualdade; g) e índice de desenvolvimento humano municipal (IDHM), indicador composto por dimensões de longevidade, educação e renda.

Importante mencionar que a iniquidade é uma medida de desigualdade sistemática e evitável, sendo injusta por não promover a tentativa de oferecer à população uma igualdade de oportunidades (17). Nesse aspecto, alguns dos indicadores utilizados avaliam, para além da riqueza, esse aspecto de baixa equidade, como o índice de Gini, o índice de Theil-L e a razão de renda.

Optou-se por utilizar a distância euclidiana como medida de similaridade em relação aos indicadores utilizados (18).

A informação sobre população coberta pela ESF considera a cobertura populacional estimada pelas equipes de saúde da família. Foi selecionado o período de 2007 a 2016. Esse período corresponde à série histórica imediatamente posterior à formulação da primeira versão da PNAB (7) e inclui ainda o ano da reformulação da atenção básica, com a publicação de nova portaria (11). Dessa forma, é possível avaliar a teoria da equidade reversa no contexto de uma política pública. Já os dados utilizados para a construção dos indicadores corresponderam ao ano de 2010, considerando a maior precisão da informação (por se tratar de fonte censitária), bem como por se tratar do ano médio do período. 
Uma vez construídos, os agrupamentos foram caracterizados com relação aos indicadores que os geraram, de forma a classificá-los como tendo situação econômica mais ou menos favorável. Para isso, foram comparadas as médias e desvio padrão de cada indicador entre os grupos. Utilizou-se o teste de ANOVA para avaliar a significância estatística da diferença entre as médias de cada grupo.

A fim de caracterizar a cobertura da ESF em cada grupo, foram calculadas as medidas de tendência central, bem como a distância interquartílica, da cobertura da ESF para cada ano da série histórica. Para a caracterização dos grupos obtidos, considerou-se a média dos indicadores utilizados na sua construção. Para que fosse possível observar a variabilidade intragrupo, obteve-se ainda o valor do desvio padrão dos indicadores, bem como o intervalo de confiança de 95\% (IC95\%) para a sua distribuição. Avaliou-se a diferença de cobertura média entre os grupos para todos os anos de observação, com o respectivo IC.

A distância interquartílica foi utilizada para avaliar o grau de homogeneidade dos grupos. Através dela, foi possível observar diferenças na cobertura da ESF no primeiro e no terceiro quartil da distribuição. Quanto menor a diferença, menor a variabilidade intragrupo.

Adicionalmente, foi realizada análise de série temporal da cobertura para os dois grupos através de regressão polinomial. A variável tempo foi centralizada no ponto médio da série histórica (19). Foram testados modelos de regressão linear simples $\left(\mathrm{Y}=\beta_{0}+\beta_{1} \mathrm{X}\right)$ e, em seguida, modelos polinomiais de segundo $\left(Y=\beta_{0}+\beta_{1} X+\beta_{2} X^{2}\right)$ e terceiro graus $\left(Y=\beta_{0}+\beta_{1} X+\beta_{2} X^{2}+\beta_{3} X^{3}\right)$. A escolha do melhor modelo se baseou no nível de significância (valor de $P$ ); no melhor ajuste, através do coeficiente de determinação $\left(\mathrm{R}^{2}\right)$; e na análise de resíduos.
A significância estatística do modelo de tendência foi admitida ao se obter $P<0,05$. Consideraram-se, como desfechos para a regressão, a cobertura pela ESF e a distância interquartílica da cobertura para cada grupo. Foi, ainda, medida a correlação entre os indicadores que deram origem aos clusters e as taxas de cobertura da ESF em cada grupo, no ano inicial (2007) e final (2016) da série histórica.

A análise foi realizada com o software $\mathrm{R}$ (versão 3.4.2), através dos pacotes cluster e timeSeries. Conforme a resolução 466/2012 do Conselho Nacional de Ética em Pesquisa (CONEP) no Brasil, o estudo está isento de aprovação por comitê de ética por se tratar de estudo com dados secundários, agregados, não nominais, de fonte pública de acesso.

\section{RESULTADOS}

A aplicação da análise de clusters, com o método two-step, ao conjunto de 5564 municípios brasileiros, gerou, a partir da estratificação conforme os sete indicadores sociais escolhidos, dois agrupamentos. A tabela 1 sintetiza a média dos escores dos indicadores para cada grupo. O grupo 1 pode ser classificado como aquele de condições menos favoráveis: possui, comparado ao outro grupo, maior mortalidade infantil, maior razão de dependência, maiores valores dos indicadores de desigualdade de renda (índice de Gini, Theil-L e razão de renda 10/40), maior proporção de pessoas vulneráveis à pobreza e menor IDHM. Ao contrário, portanto, o grupo 2 pode ser descrito como tendo condições mais favoráveis. Ressalta-se que a diferença entre os grupos, para todos os indicadores avaliados, foi estatisticamente significativa $(P<0,001)$.

A partir dos grupos já constituídos, observou-se diferença na taxa de cobertura média dos grupos ao longo dos anos (figura 1A), bem como diferença de médias entre os grupos no mesmo período (figura 1B). Em geral, independentemente do grupo, houve aumento na cobertura de APS ao longo do período. Contudo, como se pode observar, houve uma mudança, a partir de 2009, na relação entre as coberturas. Até aquele ano, a cobertura era maior no grupo com condições mais favoráveis. Ressalta-se, contudo, que desde 2007 a diferença entre os grupos (cobertura média do grupo 1 - cobertura média do grupo 2) veio caindo gradativamente, até que se inverteu, mantendo-se assim (e tornando-se, inclusive, mais evidente) até o final da série histórica. Portanto, desde 2009 a cobertura média de APS foi maior no grupo com condições menos favoráveis do que no grupo de condições mais favoráveis. Analisando a diferença de médias, constata-se que, em termos absolutos, não houve variação entre o primeiro e último ano da série, em valores absolutos - ou seja, não variou a diferença absoluta na cobertura média dos grupos de condições mais e menos favorável. No entanto, é evidente que essa diferença mudou de sentido (ou seja, a diferença relativa entre grupo 1 e 2 passou de positiva a negativa), passando a maior cobertura ao grupo com condições menos favoráveis.

A figura 2 destaca a tendência temporal dos dois indicadores utilizados para avaliar mudanças na série histórica: a cobertura (figura 2A) e a distância interquartílica (figura 2B). Observa-se, corroborando a análise anterior, uma tendência ao aumento da cobertura em ambos os grupos. Percebe-se, entretanto, que o aumento é mais destacado no grupo de municípios com condições menos favoráveis, como mostram o coeficiente angular da reta (padrão) e o coeficiente linear (nível). Através deles, vê-se que o nível do grupo 1 era menor no início da

TABELA 1. Caracterização dos agrupamentos de municípios segundo indicadores sociais selecionados, Brasil, 2007 a 2016

\begin{tabular}{|c|c|c|c|c|c|c|c|c|c|c|c|}
\hline \multirow[t]{2}{*}{ Indicador } & \multicolumn{3}{|c|}{$\begin{array}{c}\text { Grupo } 1 \\
(n=3293)\end{array}$} & \multicolumn{3}{|c|}{$\begin{array}{c}\text { Grupo } 2 \\
(n=2271)\end{array}$} & \multicolumn{3}{|c|}{$\begin{array}{c}\text { Total } \\
(n=5564)\end{array}$} & \multirow[t]{2}{*}{ Z } & \multirow[t]{2}{*}{$P$ valor } \\
\hline & Média & $\mathrm{DP}$ & IC 95\% & Média & $\mathrm{DP}$ & IC 95\% & Média & $\mathrm{DP}$ & IC 95\% & & \\
\hline Mortalidade infantil & 25,99 & 6,05 & $14,14-37,84$ & 14,57 & 2,73 & $9,22-19,91$ & 19,25 & 7,14 & $5,26-33,23$ & 9072,38 & $<0,001$ \\
\hline Razão de dependência & 59,53 & 7,73 & $44,38-74,69$ & 45,90 & 4,25 & $37,58-54,22$ & 51,49 & 8,95 & $33,95-69,03$ & 7121,96 & $<0,001$ \\
\hline Índice de Gini & 0,54 & 0,06 & $0,43-0,64$ & 0,46 & 0,06 & $0,35-0,58$ & 0,494 & 0,066 & $0,365-0,624$ & 2176,24 & $<0,001$ \\
\hline \% de vulneráveis à pobreza & 67,17 & 8,79 & $49,94-84,39$ & 27,90 & 4,95 & $18,21-37,60$ & 43,99 & 6,44 & $31,37-56,61$ & 15884,43 & $<0,001$ \\
\hline Razão $10 \%$ mais ricos $/ 40 \%$ mais pobres & 19,15 & 2,24 & $14,75-23,54$ & 11,15 & 3,59 & $4,11-18,18$ & 14,42 & 3,19 & $8,17-20,68$ & 1248,08 & $<0,001$ \\
\hline Índice de Theil - L & 0,54 & 0,11 & $0,32-0,77$ & 0,39 & 0,10 & $0,19-0,59$ & 0,46 & 0,13 & $0,20-0,71$ & 2594,34 & $<0,001$ \\
\hline IDHM & 0,59 & 0,04 & $0,51-0,67$ & 0,71 & 0,04 & $0,62-0,79$ & 0,66 & 0,07 & $0,52-0,80$ & 11176,82 & $<0,001$ \\
\hline
\end{tabular}

Fonte: IBGE, DATASUS. 
FIGURA 1. Cobertura média (A) por atenção primária à saúde e diferença de médias (B) entre grupos de municípios, Brasil, 2007 a $2016^{a}$

(A)

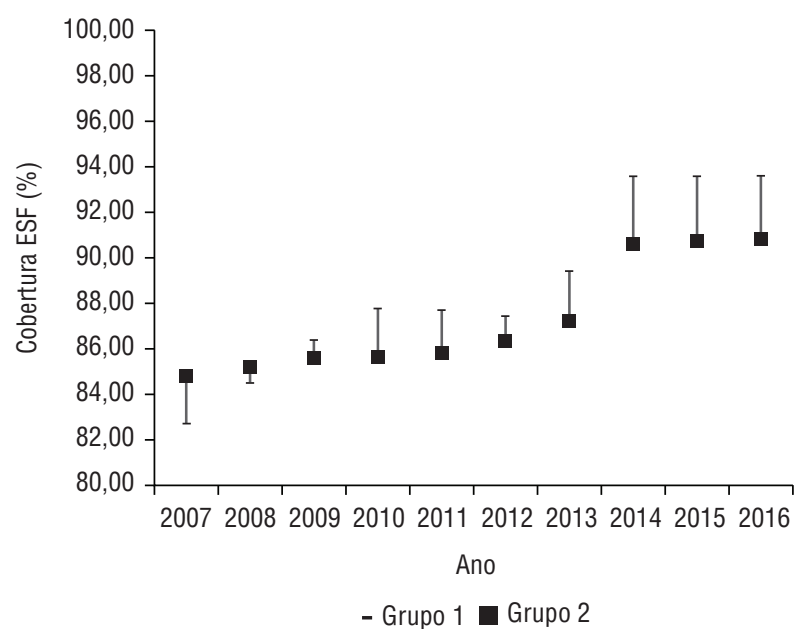

(B)

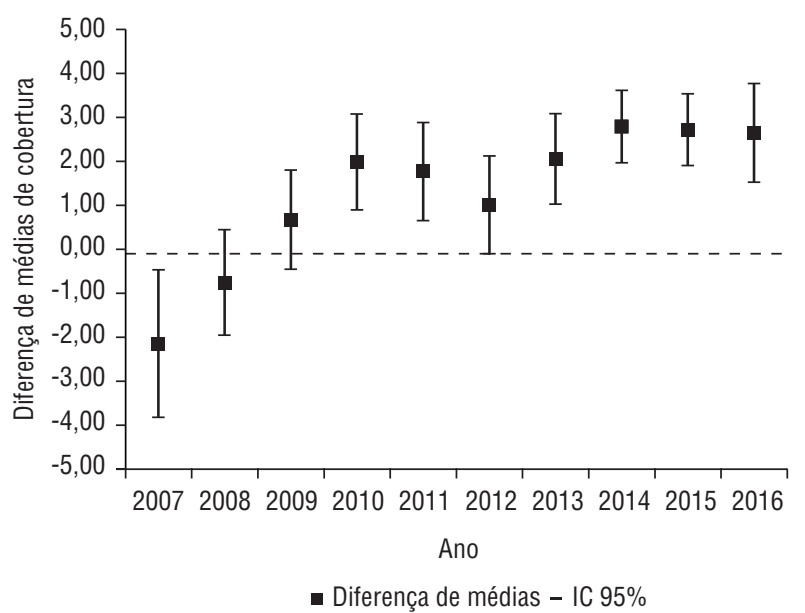

Fonte: IBGE, DATASUS.

a Grupo 1: mais vulneráveis; grupo 2: menos vulneráveis.

série, tornando-se maior ao final; ainda, tem-se que a velocidade de aumento da cobertura é maior no grupo 1.

Os grupos possuem grande número de municípios, muitos deles com cobertura de $100 \%$ pela ESF. Nesse sentido, a avaliação da diferença interquartílica, ou seja, da diferença entre o primeiro e o terceiro quartil, é um indicador adequado para avaliar o grau de dispersão dos dados em torno das medidas de tendência central. É possível, com isso, entender o comportamento dos dados de forma mais precisa. Nesse sentido, o que se observa é que a distância interquartílica, ao contrário da cobertura, vem decrescendo ao longo do período, o que significa uma tendência à homogeneidade entre os municípios com relação à cobertura. Novamente, é importante destacar a diferença entre os grupos: há, para o mesmo período, um aumento da discrepância entre eles, o que ratifica o fato de que o aumento da cobertura não tem se dado na mesma velocidade nos dois clusters, favorecendo o cluster com municípios de condições menos favoráveis.

Finalmente, independentemente do grupo, observa-se que os indicadores selecionados são correlacionados e, à exceção do IDHM, que é inverso, a correlação é direta (tabela 2). Para o grupo 1, houve mudança na correlação entre os indicadores e a cobertura pela ESF no primeiro e no último ano da série histórica. A correlação direta entre a cobertura e os indicadores sociais se tornou mais forte para mortalidade infantil, razão de dependência e proporção de vulneráveis à pobreza. Houve enfraquecimento da correlação (inversa) entre cobertura e indicadores de desigualdade de renda e fortalecimento de correlação (igualmente inversa) entre cobertura e IDHM. Isso sugere uma mudança nos critérios, ao longo dos anos, para a expansão da cobertura, valorizando especialmente a pobreza absoluta, e não a iniquidade, para a seleção de municípios prioritários. No grupo 2 houve uma redução na força de correlação (direta) com a mortalidade infantil. Destaca-se que o sentido da correlação aqui é inverso àquele observado no grupo anterior. Ainda, a correlação entre a cobertura e a proporção de vulneráveis à pobreza não foi significativa em nenhum dos anos da análise; para os indicadores de desigualdade de renda, a correlação foi inversamente proporcional à cobertura e houve igualmente uma redução na forma de correlação. Importante ressaltar que, embora significativas, algumas correlações são fracas. Esse efeito possivelmente se dá pelo grande número de municípios, e não pela força de associação em si.

\section{DISCUSSÃO}

No período do estudo houve, inequivocamente, um aumento na alocação de recursos municipais em ações e serviços públicos de saúde (5). Isso demonstra a expansão da ESF no nível mais descentralizado. No entanto, a despeito de todo o arcabouço jurídico-institucional no sentido de melhorar o atributo do acesso ao serviço, persistem algumas barreiras na concretização da APS no Sistema Único de Saúde (SUS) no Brasil (20). Uchôa et al. (21) analisaram a influência do contexto (medida através de indicadores de renda, acesso à saúde suplementar e a programas de redistribuição de renda e densidade demográfica) no desempenho dos municípios para avaliar o potencial da APS. Esses dados sugerem uma relação direta do efeito contextual com processos de territorialização, coordenação do cuidado, integralidade e oferta. Portanto, é importante qualificar a APS nesses atributos, o que exige, sobretudo, uma mudança na proposta de organização dos serviços, de modo a compreender as necessidades de saúde da população adscrita de forma localizada no tempo e no espaço de produção do cuidado $(22,23)$. A literatura mostra que, dentre os principais entraves para o pleno alcance da APS, estão o subfinanciamento e a baixa cobertura $(20,21)$. É preciso, pois, tornar real a premissa de prioridade sanitária conferida à APS, com maior investimento na estrutura e na organização da oferta de serviços.

A teoria da equidade reversa afirma que novas políticas de saúde geram desigualdade num primeiro momento e atenuam a desigualdade ao longo do tempo. A esse respeito, vale mencionar que os 
FIGURA 2. Tendência temporal da cobertura média pela atenção primária à saúde (A) e dispersão em relação à média da cobertura entre grupos (B), Brasil, 2007 a 2016 ${ }^{\mathrm{a}}$

(A)

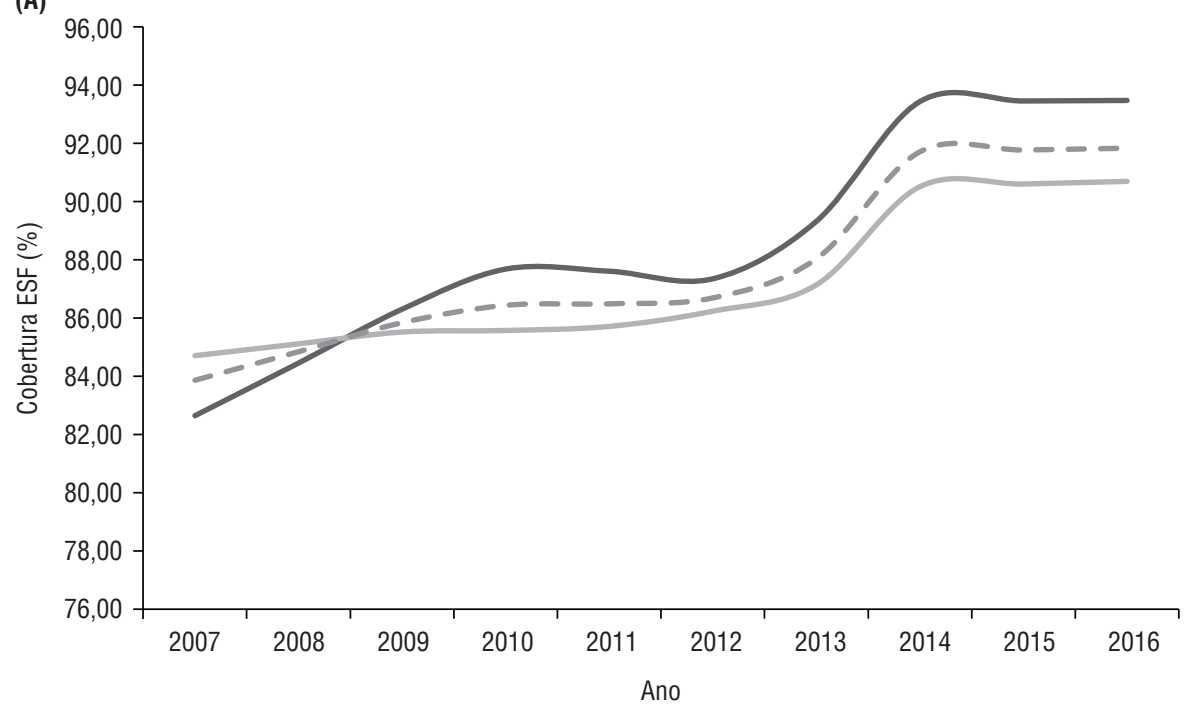

_ Grupo 1 - Grupo 2 - - - Brasil

\begin{tabular}{|c|c|c|c|c|}
\hline Grupo & Equação & $\mathrm{R}^{2}$ & $P$ valor & Tendência \\
\hline 1 & $\mathrm{y}=81,96+1,24 \mathrm{x}$ & 0,92 & $<0,001$ & Crescente \\
\hline 2 & $\mathrm{y}=83,20+0,75 \mathrm{x}$ & 0,84 & $<0,001$ & Crescente \\
\hline
\end{tabular}

(B)

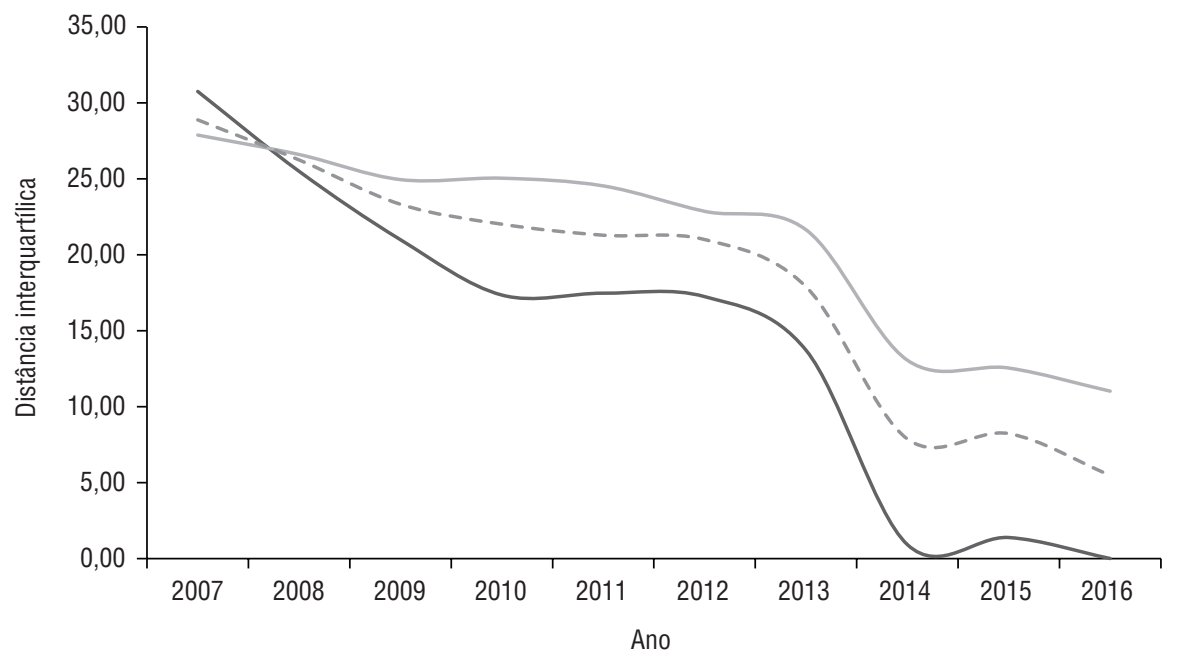

- Grupo 1 Grupo 2 - - Brasil

\begin{tabular}{|c|c|c|c|c|}
\hline Grupo & Equação & $\mathrm{R}^{2}$ & $P$ valor & Tendência \\
\hline 1 & $\mathrm{y}=33,22-3,39 \mathrm{x}$ & 0,93 & $<0,001$ & Decrescente \\
\hline 2 & $\mathrm{y}=31,85-1,96 \mathrm{x}$ & 0,87 & $<0,001$ & Decrescente \\
\hline
\end{tabular}

Fonte: IBGE, DATASUS.

a Grupo 1: mais vulneráveis; grupo 2: menos vulneráveis.

novos programas e intervenções de saúde pública alcançam inicialmente as pessoas de nível socioeconômico maior, aumentando as iniquidades entre mais ricos e mais pobres (12). Isso significa que a iniquidade somente é minimizada quando o acesso aos serviços de saúde torna-se maior entre os menos favorecidos socioeconomicamente. Cabe destacar que, mesmo em ações de saúde pública direcionada às populações mais vulneráveis, é difícil conseguir uma diminuição das iniquidades se a população de nível socioeconômico maior ainda não tiver alcançado baixos níveis de mortalidade e morbidade (24).

Há evidências de que o Brasil tem apresentado melhoria das condições sociais e econômicas da população ao longo dos últimos 20 anos $(24,25)$. Isso se deve à implementação de políticas em âmbito macroestrutural, com programas de transferência de renda como, por exemplo, o Bolsa Família $(25,26)$. Ainda assim, as desigualdades sociais são persistentes, sugerindo não ser incomum uma redução na desigualdade absoluta com aumento na desigualdade relativa fenômeno observado quando há redução nos indicadores de desigualdade, porém com menor velocidade nas camadas de menor renda $(27,28)$. Todavia, o presente estudo mostrou que a cobertura pela ESF no Brasil não confirma essa teoria.

É preciso, ainda, caracterizar a operacionalização do conceito de equidade para compreender as razões pelas quais a experiência brasileira não se encaixa na teoria da equidade reversa. A literatura tem descrito esse princípio em duas principais dimensões: condições de saúde e acesso e utilização dos serviços de saúde. Ambos se referem, em grande medida, à determinação social da saúde (29). Se, por um lado, as iniquidades acabam por expor a população vulnerável de forma diferenciada a fatores de risco para doenças, sejam elas infecciosas ou não transmissíveis, por outro lado, o acesso aos serviços de saúde determina o acolhimento da demanda dessa população e impacta diretamente na capacidade resolutiva dos serviços. Nesse sentido, a promoção da saúde, que é o paradigma adotado pela ESF, possui um conjunto de estratégias para aumentar o nível de saúde e reduzir desigualdades (30).

Outro aspecto não menos relevante é que há, na história atual do Brasil, tensões crescentes entre um projeto democratizante de saúde e um projeto neoliberal que torna o Estado mínimo, sem qualquer compromisso com a efetivação de direitos, inclusive à saúde (31). Esse ponto relativiza a capacidade da ESF de ser protagonista na articulação entre gestores e sociedade civil na construção de um projeto político que resulte em melhorias e que atenda às necessidades de saúde da população (32). Face ao desmonte progressivo que o setor saúde, no bojo de outras instâncias democráticas, vem sofrendo nos últimos meses, há 
TABELA 2. Correlação entre indicadores sociais selecionados e cobertura pela Estratégia Saúde da Família, Brasil, 2007 a 2016 a

\begin{tabular}{|c|c|c|c|c|c|c|c|c|c|c|c|}
\hline & Indicador soc & & $\begin{array}{l}\text { Mortalidade } \\
\text { infantil }\end{array}$ & $\begin{array}{c}\text { Razão de } \\
\text { dependência }\end{array}$ & $\begin{array}{l}\text { Índice de } \\
\text { Gini }\end{array}$ & $\begin{array}{c}\% \text { de } \\
\text { vulneráveis }\end{array}$ & $\begin{array}{l}\text { Razão } 10 \%+ \\
\text { ricos / } 40 \%+\end{array}$ & $\begin{array}{l}\text { Índice de } \\
\text { Theil - L }\end{array}$ & IDHM & cobESF2007 & cobESF2016 \\
\hline Cluster 1 & Mortalidade infantil & $r$ & 1 & 0,399 & 0,558 & 0,623 & 0,124 & 0,469 & $-0,615$ & 0,094 & 0,111 \\
\hline & & $P$ valor & & $<0,001$ & 0,001 & $<0,001$ & $<0,001$ & $<0,001$ & $<0,001$ & $<0,001$ & $<0,001$ \\
\hline & Razão de & $r$ & & 1 & 0,239 & 0,680 & 0,403 & 0,258 & $-0,651$ & 0,105 & 0,166 \\
\hline & dependência & $P$ valor & & & $<0,001$ & $<0,001$ & $<0,001$ & $<0,001$ & $<0,001$ & $<0,001$ & $<0,001$ \\
\hline & Índice de Gini & $r$ & & & 1 & 0,322 & 0,643 & 0,977 & $-0,019$ & $-0,189$ & $-0,165$ \\
\hline & & $P$ valor & & & & $<0,001$ & $<0,001$ & $<0,001$ & 0,272 & $<0,001$ & $<0,001$ \\
\hline & \% de vulneráveis à & $r$ & & & & 1 & 0,285 & 0,330 & $-0,825$ & 0,106 & 0,150 \\
\hline & pobreza & $P$ valor & & & & & $<0,001$ & $<0,001$ & $<0,001$ & $<0,001$ & $<0,001$ \\
\hline & Razão $10 \%$ + ricos / & $r$ & & & & & 1 & 0,676 & $-0,151$ & $-0,110$ & $-0,076$ \\
\hline & $40 \%+$ pobres & $P$ valor & & & & & & $<0,001$ & $<0,001$ & $<0,001$ & $<0,001$ \\
\hline & Índice de Theil - L & $r$ & & & & & & 1 & $-0,038$ & $-0,168$ & $-0,146$ \\
\hline & & $P$ valor & & & & & & & 0,030 & $<0,001$ & $<0,001$ \\
\hline & IDHM & r & & & & & & & 1 & $-0,188$ & $-0,247$ \\
\hline & & $P$ valor & & & & & & & & $<0,001$ & $<0,001$ \\
\hline & cobESF2007 & $r$ & & & & & & & & 1 & 0,565 \\
\hline & & $P$ valor & & & & & & & & & $<0,001$ \\
\hline & cobESF2016 & $r$ & & & & & & & & & 1 \\
\hline & & $P$ valor & & & & & & & & & \\
\hline Cluster 2 & Mortalidade infantil & $r$ & 1 & & & & & & & & \\
\hline$(n=2271)$ & & $P$ valor & & & & & & & & & \\
\hline & Razão de & $r$ & 0,166 & 1 & & & & & & & \\
\hline & dependência & $P$ valor & $<0,001$ & & & & & & & & \\
\hline & Índice de Gini & r & $-0,122$ & 0,370 & 1 & & & & & & \\
\hline & & $P$ valor & $<0,001$ & $<0,001$ & & & & & & & \\
\hline & \% de vulneráveis à & r & 0,451 & 0,559 & 0,155 & 1 & & & & & \\
\hline & pobreza & $P$ valor & $<0,001$ & $<0,001$ & $<0,001$ & & & & & & \\
\hline & Razão $10 \%$ + ricos / & r & $-0,062$ & 0,450 & 0,865 & 0,261 & 1 & & & & \\
\hline & $40 \%+$ pobres & $P$ valor & 0,003 & $<0,001$ & $<0,001$ & $<0,001$ & & & & & \\
\hline & Índice de Theil - L & $r$ & $-0,104$ & 0,288 & 0,949 & 0,115 & 0,827 & 1 & & & \\
\hline & & $P$ valor & $<0,001$ & $<0,001$ & $<0,001$ & $<0,001$ & $<0,001$ & & & & \\
\hline & IDHM & $r$ & $-0,549$ & $-0,466$ & 0,012 & $-0,762$ & $-0,153$ & 0,063 & 1 & & \\
\hline & & $P$ valor & $<0,001$ & $<0,001$ & 0,574 & $<0,001$ & $<0,001$ & 0,003 & & & \\
\hline & cobESF2007 & $r$ & 0,122 & $-0,178$ & $-0,239$ & $-0,041$ & $-0,226$ & $-0,166$ & 0,054 & 1 & \\
\hline & & $P$ valor & $<0,001$ & $<0,001$ & $<0,001$ & 0,059 & $<0,001$ & $<0,001$ & 0,009 & & \\
\hline & cobESF2016 & $r$ & 0,090 & $-0,163$ & $-0,194$ & 0,002 & $-0,194$ & $-0,134$ & 0,036 & 0,513 & 1 \\
\hline & & $P$ valor & $<0,001$ & $<0,001$ & $<0,001$ & 0,930 & $<0,001$ & $<0,001$ & 0,088 & $<0,001$ & \\
\hline
\end{tabular}

a Grupo 1: mais vulneráveis; grupo 2: menos vulneráveis.

uma tendência de conversão integral dos direitos sociais à lógica de mercado. Percebe-se, hoje, uma correlação de forças desfavorável às instituições e recursos sociais que defendem a saúde como um direito (33).

Desde 2017, há uma nova PNAB no Brasil, que deve ser analisada com cautela pela ameaça de fazer retornar uma concepção de atenção primária seletiva, limitada e que vai de encontro à proposta de universalidade e integralidade, princípios doutrinários sobre os quais a ESF foi construída (10). Isso pode agravar as desigualdades mencionadas na análise realizada neste estudo, uma vez que impõe retrocessos para a construção de uma APS integral: as normativas relativizam a cobertura populacional da ESF; segmentam o cuidado a certos padrões assistenciais; reposicionam a ESF no contexto da APS, retornando ao formato da APS tradicional; e descaracterizam o perfil do agente comunitário de saúde.

Em razão dessa realidade, é importante que haja um esforço no sentido de reunir toda a evidência científica que destaque as conquistas da APS, fruto de uma construção histórica num contexto de marcadas desigualdades (34). É necessário que a possibilidade de ter sido criada e expandida, procurando atender ao princípio da equidade - e, portanto, fugindo da regra observada pela teoria da equidade reversa - seja avaliada e divulgada.

O estudo possui limitações: primeiramente, é importante notar que a disponibilidade do serviço, per se, não garante o acesso, uma vez que há fatores relacionados ao consumo e à oferta dos serviços que precisam ser considerados, como o estabelecimento de redes de serviços (que tomam a APS como porta de entrada) e grau de satisfação dos usuários, entre outros (35-37). Ainda, o uso do nível municipal como escala de 
avaliação não dá conta, por exemplo, da desigualdade na distribuição espacial das unidades de saúde, com concentração em bairros ou distritos de condições de vida mais ou menos favoráveis. De fato, o indicador utilizado considera o número de equipes. Assim, para cobrir a maioria ou totalidade da população de municípios menores, poucas equipes são suficientes. Isso não se traduz diretamente em aumento de acesso, quando consideradas iniquidades intramunicipais, em especial em situações de vulnerabilidade, como em áreas rurais ou

Além disso, dada a indisponibilidade dos dados anuais para os indicadores sociais, foram utilizados dados referentes ao ano do meio do período que, afortunadamente, trata-se de ano censitário, violentas.

o que garante a qualidade dos dados. Não foi possível, por conta disso, observar a redução consistente da pobreza e da extrema pobreza no período, o que permite, por exemplo, o crescimento do setor privado na provisão de seguro de saúde. Salienta-se, contudo, que são questões que não necessariamente impactam no aumento da cobertura da ESF. Conforme dito, é necessário fazer uma distinção entre cobertura e acesso, pois se tratam de diferentes atributos, e um não é consequência direta do outro. Como força do estudo, pode-se dizer que o fato de trabalhar com dados de quase 6000 municípios demonstra a robustez da análise, uma vez que foi possível avaliar o contexto de um país de dimensões continentais, com marcada desigualdade.

\section{REFERÊNCIAS}

1. Prates ML, Machado JC, Silva LS, Avelar PS, Prates LL, Mendonça ET, et al. Desempenho da Atenção Primária à Saúde segundo o instrumento PCATool: uma revisão sistemática. Cienc Saude Coletiva. 2017;22(6):1881-93.

2. Paula WKAS, Samico IC, Caminha MFC, Batista Filho M, Silva SL. Primary health care assessment from the users' perspectives: a systematic review. Rev Esc Enferm USP. 2016;50(2):331-40.

3. Machado CV, Baptista TWF, Nogueira CO. Políticas de saúde no Brasil nos anos 2000: a agenda federal de prioridades. Cad Saude Publica. 2011;27(3):521-32.

4. Viana ALD, Dal Poz MR. A reforma do sistema de saúde do Brasil e o Programa Saúde da Família. Physis. 1998;8(2):11-48.

5. Costa NR.A Estratégia de Saúde da Família, a atenção primária e o desafio das metrópoles brasileiras. Cienc Saude Colet. 2016;21(5):1389-98.

6. Domingos CM, Nunes EFPA, Carvalho BG, Mendonça FF. A legislação da atenção básica do Sistema Único de Saúde: uma análise documental. Cad Saude Publica. 2016;32(3):e00181314.

7. Brasil, Ministério da Saúde. Política nacional de atenção básica. Brasília: Ministério da Saúde; 2006. Disponível em: http:// bvsms.saude.gov.br/bvs/publicacoes/politica_nacional_atencao_basica_2006.pdf Acessado em agosto de 2018.

8. Castro ALB, Machado CV. A política federal de atenção básica à saúde no Brasil nos anos 2000. Physis. 2012;22:477-506.

9. Campos RTO, Ferrer AL, Gama CAP, Campos GWS, Trapé TL, Dantas DV. Avaliação da qualidade do acesso na atenção primária de uma grande cidade brasileira na perspectiva dos usuários. Saude Debate. 2014;38(spe):252-64.

10. Morosini MVGC, Fonseca AF, Lima LD. Política Nacional de Atenção Básica 2017: retrocessos e riscos para o Sistema Único de Saúde. Saude Debate. 2018;42(116):11-24.

11. Fontenelle LF. Mudanças recentes na Política Nacional de Atenção Básica: uma análise crítica. Rev Bras Med Fam Comunidade. 2012;7(22):5-9.

12. Victora CG, Vaughan JP, Barros FC, Silva $\mathrm{AC}$, Tomasi E. Explaining trends in inequities: evidence from Brazilian child health studies. Lancet. 2000;356:1093-98.

13. Instituto Brasileiro de Geografia e Estatística (IBGE). Censos demográficos. Indicadores sociais municipais: uma análise dos resultados do universo do Censo Demográfico. Disponível em: https://ww2.ibge.gov.br/ home/estatistica/populacao/censo2010/ indicadores_sociais_municipais/default indicadores_sociais_municipais.shtm Acessado em agosto de 2018.

14. Hair JF, Black WC, Babin BJ, Anderson RE, Tatham RL. Análise multivariada de dados. $6^{\mathrm{a}}$ ed. Porto Alegre: Bookman; 2009.

15. Yoshimitanaka O, Drumond Jr M, Cristo EB, Spedo SM, Pinto NR. Uso da análise de clusters como ferramenta de apoio à gestão no SUS. Saude Soc. 2015;24(1):34-45.

16. Figueiredo Filho DB, Rocha EC, Silva MB, Paranhos R, Silva Jr JA, Duarte BSF. Cluster analysis for political scientists. Appl Math. 2014;5:2408-15.

17. Buss PM, Pellegrini Filho A. Iniqüidades em saúde no Brasil, nossa mais grave doença: comentários sobre o documento de referência e os trabalhos da Comissão Nacional sobre Determinantes Sociais da Saúde. Cad Saude Publica. 2006;22(9),2005-8.

18. Figueiredo Filho DB, Silva Jr JA, Santos Filho RP, Rocha EC, Nascimento WS, Silva análise fatorial e análise de cluster paras mensurar a qualidade das políticas públicas. Teor Soc. 2014;22(2):123-52.

19. Kleinbaum DG, Kupper LL, Muller KE. Applied regression analysis and other $\mathrm{MB}$, et al. Happy together: como utilizar
Finalmente, a conclusão é de que a expansão da cobertura é uma categoria analítica importante para apreender as iniquidades em saúde, e de que a descrição da tendência da cobertura, à luz da teoria da equidade reversa, comprova isso.

Agradecimentos. A Luiz Inácio Lula da Silva, por seu legado na tentativa de reduzir a desigualdade social no Brasil.

Conflitos de interesse. Nada declarado pelo autor.

Declaração. As opiniões expressas no manuscrito são de responsabilidade exclusiva dos autores e não refletem necessariamente a opinião ou política da RPSP / PAJPH ou da Organização Pan-Americana da Saúde (OPAS). multivariable methods. $2^{\mathrm{a}}$ ed. Belmont: Duxbury Press; 1988.

20. Lima SAV, Silva MRF, Carvalho EMF, Pessoa EAC, Brito ESV, Braga JPR. Elementos que influenciam o acesso à atenção primária na perspectiva dos profissionais e dos usuários de uma rede de serviços de saúde do Recife. Physis. 2015;25(2); 635-56.

21. Uchôa SAC, Arcêncio RA, Fronteira ISE, Coêlho AA, Martiniano CS, Brandão ICA et al. Acesso potencial à atenção primária em saúde: o que mostram os dados do programa de melhoria do acesso e da qualidade do Brasil? Rev Latino-Am Enfermagem. 2016;24:e2672.

22. Lavras C. Atenção primária à saúde e a organização de redes regionais de atenção à saúde no Brasil. Saude Soc.2011; 20(4): 867-74.

23. Starfield B. Atenção primária: equilíbrio entre necessidades de saúde, serviços e tecnologia. Brasília: UNESCO, Ministério da Saúde; 2002. Disponível em: http://bvsms.saude.gov.br/bvs/publicacoes/atencao_primaria_p1.pdf Acessado em agosto de 2018.

24. Albuquerque MV, Viana ALA, Lima LD, Ferreira MP, Fusaro ER, Iozzi FL. Desigualdades regionais na saúde: mudanças observadas no Brasil de 2000 a 2016. Cienc Saude Coletiva. 2017;22(4);1055-64.

25. Arretche M, org.Trajetórias da desigualdade: como o Brasil mudou nos últimos 50 anos. São Paulo: Editora UNESP; 2015.

26. Pega F, Liu SY, Walter S, Pabayo R, Saith R, Lhachimi SK. Unconditional cash transfers for reducing poverty and vulnerabilities: effect on use ofhealth services andhealthoutcomes in low-and middle-income countries. Cochrane Database Syst Rev. 2017;15(11):CD011135.

27. Escoda MSQ. Sobre iniquidade em saúde. Rev Espaço Saude. 2004;6:16-24. 
28. Santos AMA, Jacinto PDA, Tejada CAO. Causalidade entre renda e Saúde: uma análise através da abordagem de dados em painel com os estados do Brasil. Est Econ. 2012;42(2):229-61.

29. Escorel S. Os dilemas da equidade em saúde: aspectos conceituais. Brasília, DF: OPAS; 2001.

30. Oliveira RTQ, Ignacio CF, Moraes Neto AHA, Barata MML. Matriz de avaliação de programas de promoção da saúde em territórios de vulnerabilidade social. Cienc Saúde Coletiva. 2017;22(12):3915-932.

31. Silva HF, França Jr I. A combinação de projetos políticos distintos na atenção básica à saúde: impactos político-sociais e a desconstrução do SUS. Physis. 2015;25(2): 423-42.
32. Morimoto T, Costa JSD. Internações por condições sensíveis à atenção primária, gastos com saúde e Estratégia Saúde da Família: uma análise de tendência. Cienc Saude Coletiva. 2017;22(3):891-900.

33. Morosini MVGC, Fonseca AF. Reviewing the Brazilian National Primary Health Care Policy at such a time?Cad Saude Publica. 2017;33(1):e00206316.

34. Orlandin EAS, Moscovici L, Franzon ACA, Passos ADC, Fabbro ALD, Vieira EM, et al. Uma agenda de pesquisa para a Atenção Primária à Saúde no estado de São Paulo, Brasil: o estudo ELECT. Interface (Botucatu). 2017;21(61):349-61.

35. Barros AJD, Victora CG. Measuring coverage in $\mathrm{MNCH}$ : determining and interpreting inequalities in coverage of maternal, newborn, and child health interventions. PLoS Med. 2013;10(5):e1001390.

36. Barros FPC, Lopes JS, Mendonça AVM, Sousa MF. Acesso e equidade nos serviços de saúde: uma revisão estruturada. Saude Debate. 2016;40(110):264-71.

37. Conill EM. Ensaio histórico-conceitual sobre a Atenção Primária à Saúde: desafios para a organização de serviços básicos e da Estratégia Saúde da Família em centros urbanos no Brasil. Cad Saude Publica. 2008;24(Supl 1):7-27.

Manuscrito recebido em 2 de janeiro de 2018. Aceito em versão revisada em 24 de julho de 2018.
ABSTRACT

\section{Does the inverse theory hypothesis apply to primary health care? Evidence from 5564 Brazilian municipalities}

Keywords Primary health care; public policy; equity; health inequalities; Brazil.
Objective. To investigate the evolution of primary health care (PHC) coverage in Brazilian municipalities from 2007 to 2016 from the perspective of the inverse theory hypothesis.

Method. This ecological study was performed with data from 5564 Brazilian municipalities. Data were obtained from the Brazilian Institute of Geography and Statistics (IBGE) and from the Unified Health System database (DATASUS). The municipalities were classified according to their social development status using selected cluster indicators. After classification of the municipalities, PHC was analyzed by determining Family health Strategy (FHS) coverage through means and interquartile distance, with analysis of time series and correlations between variables.

Results. Two groups of municipalities were detected: a group with more favorable $(n=3293)$ and a group with less favorable conditions $(n=2271)$. The difference between the groups for all indicators was statistically significant $(\mathrm{P}<0.001)$. In general, regardless of the group, an increase in FHS coverage was detected along the study period. However, from 2009 on, mean FHS coverage became higher in the group with less favorable conditions. The increase in coverage was also faster in this group. Finally, a change in the correlation between indicators and PHC coverage in the first vs. last year of the historical series indicates that the criteria used to expand coverage in the group with less favorable conditions shifted to favor absolute poverty rather than inequality.

Conclusions. PHC in Brazil fulfills its role of reducing access inequalities. Therefore, the inverse theory hypothesis does not apply to this case. 
RESUMEN

¿Se aplica la hipótesis de la equidad inversa a la atención primaria de salud? Pruebas obtenidas en 5564 municipios brasileños

Objetivo. Investigar la evolución de la cobertura de la atención primaria de salud en los municipios brasileños desde el 2007 hasta el 2016 en el contexto de la hipótesis de la equidad inversa.

Métodos. Estudio ecológico realizado con datos de 5564 municipios brasileños, obtenidos a partir del censo del 2010 y de la base de datos del Sistema Único de Salud (DATASUS). Los municipios se clasificaron según su grado de desarrollo con un análisis por conglomerados, a partir de algunos indicadores sociales seleccionados. Se examinó la cobertura con la estrategia de salud de la familia en los grupos creados sobre la base de la mediana y del rango intercuartílico, con análisis de series temporales y correlación entre variables.

Resultados. Se crearon dos grupos de municipios con condiciones más favorables ( $\mathrm{n}=3$ 293) o menos favorables $(\mathrm{n}=2$ 271). Se observó una diferencia significativa $(\mathrm{P}<0,001)$ entre los grupos en todos los indicadores evaluados. En general, independientemente del grupo, aumentó la cobertura con la estrategia de salud de la familia a lo largo del período de estudio. Sin embargo, a partir del 2009, la tasa mediana de cobertura con dicha estrategia se incrementó en el grupo con condiciones menos favorables. El aumento de la cobertura también fue más acelerado en ese grupo. Finalmente, un cambio de la correlación entre los indicadores y la cobertura de la atención primaria de salud en el primero y el último año de la serie histórica indica que los criterios utilizados para la ampliación de la cobertura en el grupo con condiciones menos favorables asignaron valor a la pobreza absoluta, pero no a la inequidad.

Conclusiones. La atención primaria de salud en Brasil cumple su función como política de reducción de la desigualdad en el acceso. Por lo tanto, la hipótesis de la equidad inversa no se aplica en este caso.

Palabras clave Atención primaria de salud; políticas públicas; equidad; inequidad en salud; Brasil. 